\title{
Des dispositifs pluralistes sous contrôle : internaliser les critiques pour gouverner le stockage des déchets radioactifs ${ }^{\star}$
}

\author{
Julie Blanck* \\ Sociologie, Chaire Santé, Sciences-Po Paris, Paris, France
}

\begin{abstract}
Résumé - Le stockage géologique de déchets radioactifs constitue un cas de politisation du sous-sol, autour d'un objet hybride alliant activités minières et nucléaires. Cet article explore les transformations du gouvernement de ce stockage, avec la mise en place de trois dispositifs pluralistes de pilotage et d'internalisation de critiques, afin de désamorcer les mouvements contestataires au moment de sa réalisation. Il étudie successivement la démarche de concertation pour la sélection de la zone d'implantation; le groupe de pilotage du plan national pour la gestion des matières et déchets radioactifs et le débat public organisé en 2013 sur les modalités du stockage. À travers ces trois dispositifs, les promoteurs du projet tentent d'en maîtriser la politisation : ils intègrent ou écartent certaines critiques pour légitimer un projet contesté, tout en verrouillant son opportunité.
\end{abstract}

Mots-clés : stockage géologique / déchets radioactifs / politisation / critiques / gouvernement des risques

\begin{abstract}
Pluralist processes under control: internalizing criticism to govern the storage of radioactive waste. The geological disposal of radioactive waste offers a particularly visible case of politicization of the subsurface around a hybrid project which combines mining and nuclear activities. This article explores the changes in disposal governance involving the creation of three pluralistic systems for piloting and internalizing criticism in order to defuse protest movements at the time of its implementation. It successively studies the concertation process for the selection of the disposal area, the steering group of the National Plan for the Management of Radioactive Materials and Waste, and the Public Debate organized in 2013 on storage conditions and methods. Through these three instruments the operators attempt to set up a controlled and ambiguous process of politicization. Projects are altered to legitimate them by taking into account requests from critical actors, although the most radical criticism such as questioning the timeliness of a project is dismissed and its opportunity kept firmly out of the debate.
\end{abstract}

Keywords: geological storage / radioactive waste / politicization / criticism / risk government

Le stockage géologique de déchets radioactifs constitue un cas de politisation du sous-sol. Il s'agit d'un objet hybride, alliant activités minières et nucléaires. Jusqu'à présent, il a été étudié davantage en lien avec l'industrie électronucléaire qu'avec l'exploitation du sous-sol. Pourtant, ce rapprochement avec d'autres usages du sous-sol participe à une démarche de géologie politique. L'énergie nucléaire est présentée par ses promoteurs comme une ressource différente des ressources minières, alors qu'il s'agit d'exploiter et de

\footnotetext{
* Cet article est une contribution au numéro coordonné par X. Arnauld de Sartre et S. Chailleux, intitulé «Pour une géologie politique $»$.

*Auteur correspondant : julieblanck@gmail.com
}

transformer un minerai, l'uranium, qui sera ensuite replacé dans des stockages souterrains. Dès lors, il est possible de déconstruire ces discours de «singularisation» du nucléaire portés par les opérateurs (Hecht, 2012) et de réinscrire les activités nucléaires dans le giron d'activités liées au sous-sol.

Cet article explore les liens complexes entre contestations et transformations récentes du gouvernement du stockage géologique des déchets radioactifs, en suivant des dispositifs pluralistes de pilotage et d'internalisation de critiques: ils ont pour fonction de produire du consensus et de désamorcer les contestations. Alors que les dispositifs de participation sont habituellement étudiés de l'extérieur dans les espaces publics, je propose d'étudier de l'intérieur comment les promoteurs 
du stockage testent un processus maitrisé de politisation, aux effets limités : ces dispositifs modifient un projet en incluant les demandes d'acteurs critiques, mais ils écartent les positions radicales contre le projet. Ils donnent une plus grande légitimité politique à un projet contesté, tout en participant au maintien du choix du stockage.

Le stockage géologique est politisé en raison de fortes contestations (Barthe, 2006). Habituellement, le processus de politisation définit et publicise les caractéristiques politiques d'objets (Lagroye, 2003) : des acteurs peuvent alors requalifier comme politiques des enjeux ou des activités qui ne l'étaient pas. Ce processus peut donner lieu à une mise en politique (Barthe, 2006), pour demander la reconnaissance d'un problème comme public et une action de la part de l'État. Ce processus peut alors ouvrir des espaces de débat pour discuter de l'opportunité de projets, d'intérêts et de représentations d'acteurs variés. Depuis l'accident de Tchernobyl, le secteur nucléaire connaît un processus de démocratisation et d'assimilation des critiques (Topçu, 2013). La création des dispositifs pluralistes de concertation s'inscrit dans cette logique pour associer des acteurs variés à la définition de ce projet contesté. Cette stratégie d'ouverture a des effets sur la conception du stockage et lui donne une légitimité politique. Cependant, à travers ces dispositifs sous contraintes, les promoteurs du projet contrôlent et imposent une politisation limitée en internalisant certaines critiques compatibles et en écartant les critiques les plus radicales (qui remettent en cause l'opportunité du projet). Ces dispositifs participent donc au maintien d'un projet contesté. Plutôt qu'un processus déterministe de «dépendance au sentier», (d'auto-renforcement par le coût que représenterait tout changement d'option (Pierson, 2000)), la construction du verrouillage autour de cette solution repose davantage sur le travail d'acteurs de plus en plus nombreux qui participent à sa légitimation.

Depuis les années 1980, la politisation du stockage géologique des déchets les plus radioactifs est liée aux contestations. L'Agence nationale pour la gestion des déchets radioactifs (Andra) a été créée en 1979 comme filiale du Commissariat à l'énergie atomique (CEA) pour porter ce projet (Blanck, 2016, 2017). Entre 1984 et 1989, l'Andra prépare une reconnaissance géologique sur quatre sites, mais l'arrivée des géologues de l'Andra et du BRGM (Bureau de recherches géologiques et minières) provoque des mouvements sociaux qui empêchent les opérations. Ces mobilisations bloquent le projet sur trois sites et se coordonnent à l'échelle nationale. À la suite d'une manifestation en janvier 1990, le Gouvernement décrète un moratoire pour suspendre le projet et «prendre le temps de se parler». Ces contestations sont à l'origine d'une «mise en politique » des déchets radioactifs (Barthe, 2006): ils quittent la sphère discrète des opérateurs techniques et sont pris en charge par les parlementaires dans la sphère publique.
Pour sortir de cette crise, le Gouvernement confie ce problème à l'Office parlementaire d'évaluation des choix scientifiques et technologiques (OPECST). Dans son rapport, puis dans la loi de 1991, le député Christian Bataille réaffirme l'inéluctabilité du stockage, reprenant les arguments des opérateurs nucléaires, et propose de transformer le projet industriel en projet de recherche de 15 ans : pour construire des laboratoires souterrains sur plusieurs sites et choisir le meilleur. Parallèlement, le CEA doit étudier deux solutions, la séparation/transmutation (retraitement poussé) et l'entreposage: Yannick Barthe parle d'une réouverture des possibles et d'une « réversibilisation » de la gestion des déchets radioactifs. Mais derrière cette mise en scène publique, le choix du stockage est maintenu discrètement par les opérateurs et le Gouvernement (Blanck, 2016) : ces changements politiques doivent montrer que des critiques sont assimilées, tout en conservant l'objectif initial.

L'agence construit un seul laboratoire souterrain à Bure et produit des données géologiques pour démontrer la faisabilité et la sûreté du stockage. Une fois le dossier scientifique validé par un groupe d'experts (scientifiques et de sûreté), le stockage est retenu comme solution de référence par la loi de 2006. Dès lors, l'Andra entre dans une phase de construction du stockage : cette concrétisation cristallise de nombreux risques techniques, économiques, politiques et sociaux. Et cette controverse est de plus en plus polarisée (Borraz, 2008), opposant promoteurs et acteurs critiques dans deux camps de plus en plus clivés. Les acteurs réglementaires et les opérateurs multiplient alors les dispositifs de concertation afin de maîtriser le processus de politisation, d'internaliser certaines critiques et d'en évacuer d'autres, pour donner au stockage une plus grande légitimité politique, sans que son opportunité ne soit discutée. Ces structures organisationnelles permettent ainsi d'associer des acteurs plus nombreux, y compris critiques, au gouvernement de la gestion des déchets : à sa définition, à sa mise en auvre et à son suivi. Ces dispositifs s'inscrivent dans la continuité de démarches plus anciennes: comme les comités d'expertise pluraliste étudiés par Sezin Topçu, notamment le groupe sur l'après-mine (Topçu, 2013), la commission locale d'information et de suivi ou encore le débat public national (Blatrix et al., 2007 ; Mabi, 2013).

Dans cet article, je propose d'étudier de l'intérieur la création et l'utilisation par l'Andra de trois dispositifs de participation, qui visent à intégrer les critiques d'acteurs variés dans la conception et le gouvernement ${ }^{1}$ d'un

\footnotetext{
${ }^{1}$ À la suite de $\mathrm{S}$. Topçu, je retiens la notion de gouvernement, et non de gouvernance, car le ministère en charge de l'énergie joue un rôle central dans la définition et la mise en œuvre de cette politique publique, suivant une approche très centralisée, hiérarchique et descendante.
} 
stockage plus consensuel. Le premier dispositif est une démarche d'intéressement des acteurs locaux dans le choix d'une zone d'implantation du stockage : les acteurs sont sollicités pour formuler des préférences dans le cadre d'un processus de territorialisation. Pour étudier ce dispositif, j'ai réalisé des entretiens avec des personnes ayant participé à sa mise en place (dirigeants, agents, experts) et j'ai analysé la littérature grise (rapports, avis).

Le deuxième dispositif concerne l'ensemble de l'aval de la filière nucléaire : le groupe de travail sur le Plan national de gestion des matières et déchets radioactifs (PNGMDR). Ce groupe de pilotage pluraliste associe ministères, autorités de sûreté, opérateurs et associations environnementales pour définir et mettre en œuvre ce plan. J'ai réalisé des observations entre 2012 et 2014, assisté à des réunions restreintes et séances plénières, réalisé des entretiens avec des participants et étudié des documents publics et de la littérature grise (plusieurs versions du plan, comptes rendus, présentations).

Le troisième dispositif, le débat public national de 2013, est plus classique. Il a été l'occasion d'une forte médiatisation et " polarisation » de la controverse (Borraz, 2008), au point d'être intenable. Ses modalités ont été modifiées dans l'urgence pour le sortir de l'espace public et écarter les acteurs critiques les plus virulents. Je propose de ne pas suivre le débat à travers les échanges et l'évolution des positions de chacun (voir les études du débat de 2005 sur les déchets radioactifs par Topçu, 2013 ; Blatrix et al., 2007), mais davantage à travers sa préparation et son usage interne. Derrière ce débat « raté », qui lui échappe, l'agence s'approprie certaines critiques pour justifier des modifications du cadre politique du projet. Pour rendre compte de ce point de vue interne, j'ai observé les comités de pilotage du débat public, réunions publiques et séances du Coesdic (Comité d'expertise et de suivi de la démarche d'information et de consultation).

Ces trois dispositifs saisissent différentes formes d'assimilation de critiques dans la conception et le gouvernement du stockage géologique. En étudiant cette stratégie de systématisation de dispositifs pluralistes, la direction cherche à mettre en œuvre une démarche concertée, pour enrichir et consolider le projet. Pour cela, elle définit un cadre de ce qui est négociable ou non, afin d'éviter la remise en cause de l'opportunité du projet. L'articulation entre critiques et politisation constitue le cœur du processus de légitimation de ce projet contesté. Il ne s'agit pas non plus d'un véritable forum hybride, idéal, d'une démocratie technique (Callon et al., 2001) : alors que les dispositifs participatifs sont censés permettre de rouvrir les possibles et de discuter de l'opportunité d'un projet (Barthe, 2006), les échanges portent seulement sur les modalités de réalisation d'un stockage considéré comme inéluctable, son opportunité n'est pas négociée (Blanck, 2016).

\section{Encadré - Présentation de la méthodologie}

Bénéficiant d'une bourse de recherche de l'Andra, j'ai analysé ses archives internes organisationnelles et techniques, puis réalisé des entretiens avec des acteurs historiques sur leurs activités. Entre 2011 et 2014, j’ai mené une analyse organisationnelle de l'agence, en étudiant des documents internes variés (manuels d'organisations, notes de projets, comptes rendus de réunions, rapports), en faisant des entretiens d'activités et des observations dans des dispositifs internes et externes (comités stratégiques, comités de pilotage, réunions entre Andra et producteurs, débat public, auditions parlementaires). Au total, 61 entretiens semidirectifs ont été réalisés, ainsi que de nombreux entretiens informels. Les stratégies des acteurs critiques ont été étudiées au prisme de leurs interactions avec les promoteurs du projet de stockage, notamment lors des séances d'observation.

Cette recherche consacrée au gouvernement du temps long a été évaluée et retenue par le Groupement de laboratoires de sciences humaines et sociales auquel participent des sociologues, historiens et économistes d'institutions de recherche*. Ce groupement a été créé en 2007 pour aider l'Andra dans la préparation du débat public. Il était piloté par Luis Aparicio (docteur en sociologie des sciences) et finance des projets et thèses sur des thématiques intéressant chercheurs et opérateurs. L. Aparicio et les membres de ce groupement m'ont aidée à m'insérer dans cette organisation pour l'étudier tout en préservant mon autonomie, jouant du double statut de salariée et d'observatrice. J'ai ainsi pu définir mon projet librement, négocier l'ouverture de terrains, solliciter des entretiens, accéder à des archives et documents. Certains accès m'ont été refusés et j'ai tenté de combler ces lacunes avec des entretiens et des documents. Par ailleurs, je n'ai pas effectué de travail de conseil en sociologie auprès des membres de l'Andra: mon rôle est resté celui d'une observatrice.

J'ai échangé régulièrement avec les acteurs de manière informelle et organisé des restitutions afin de recueillir leurs réactions. Pour la rédaction de la thèse et d'articles, il n'y a eu aucune validation de la part de l'Andra, mon travail leur a été transmis une fois validé scientifiquement.

* Chercheurs du Laboratoire Interdisciplinaire Sciences Innovations Sociétés Lisis (Pierre-Benoît. Joly), du Cermes3 Centre de recherche médecine, sciences, santé, santé mentale, société (Soraya Boudia), du CSO Centre de Sociologie des Organisations (Olivier Borraz), du CSI Centre de Sociologie de l'Innovation (Michel Callon puis Madeleine Akrich), Cetcopra Centre d'étude des techniques, des connaissances et des pratiques (Bernadette Bensaude-Vincent, Sophie Poirot-Delpech et Laurence Raineau-Facchini) et du Centre Alexandre Koyré (thèse de Leny Patinaux). 


\section{Associer les acteurs locaux au choix de la zone d'implantation}

En 2006, le dossier scientifique de l'Andra a été validé par ses évaluateurs politiques, scientifiques et experts en sûreté. L'Andra est alors autorisée à préparer la construction du stockage. Cette phase de concrétisation est particulièrement sensible: à la fin des années 1980, elle avait cristallisé les tensions et favorisé les contestations. Avant fin 2009, l'Agence doit définir une zone restreinte de $30 \mathrm{~km}^{2}$ sur laquelle implanter le stockage (sur les $250 \mathrm{~km}^{2}$ étudiés) : ce choix matérialise le passage d'un projet de recherche à la réalisation industrielle. Pour favoriser l'acceptation $\mathrm{du}$ projet localement, la direction décide de mettre en œuvre une démarche de concertation et d'associer les acteurs locaux à la définition de cette zone et de réduire les risques de contestations. Pour cela, elle se fait accompagner par des professionnels et experts de la concertation.

Elle établit alors une procédure de négociation limitée à certains éléments, afin de maîtriser le processus de politisation locale et d'éviter une renégociation globale du projet, déjà très avancé. La directrice générale de l'agence explique: "On a mis tous nos efforts sur le politique et les choix d'implantation du site. Ça, c'est le plus difficile à faire ${ }^{2}$. » Au lieu de sélectionner cette zone de manière unilatérale, sur des critères techniques et scientifiques, la direction adopte une stratégie «progressive» de réduction de la taille de la zone, en plusieurs étapes ${ }^{3}$. Il s'agit de différencier ce qui relève de choix techniques (non négociables pour des raisons de sûreté ou industrielles) et de choix qui seraient politiques et sociaux, donc discutables. Le directeur des projets explique comment il a présenté cette stratégie aux acteurs : «Un premier critère, c'est la géologie, mais ce critère peut nous laisser une certaine flexibilité, donc ça nous intéresserait de recueillir votre point de vue, en nous disant où implanter ou au contraire où ne pas le mettre, et pourquoi. Et après on verra ce qu'on en fera ${ }^{4}$.» Cette démarche progressive permet d'établir un cadre et de réduire en amont l'éventail des possibles techniques, pour ne discuter que d'options modifiables.

Lors de la première étape, les géologues précisent les caractéristiques de la couche étudiée et délimitent une "zone géologique favorable» selon des critères scientifiques et de sûreté en sous-sol: absence de failles mineures, mesures sismiques, épaisseur de la couche,

\footnotetext{
2 Entretien avec la directrice générale de l'Andra, 28 avril 2013.

3 Projet de stockage géologique profond réversible: Proposition d'une zone d'intérêt pour la reconnaissance approfondie et de scénarios d'implantation en surface, Comité de Haut Niveau, 18 novembre 2009, Andra.

4 Entretien avec le directeur des projets, 29 mars 2013.
}

profondeur, gradient de charge. Cette étape justifie scientifiquement le choix d'une zone élargie, avant de la préciser selon d'autres critères. Avec la deuxième étape, les ingénieurs en charge de la conception et de la maîtrise des risques réduisent la zone à $80 \mathrm{~km}^{2}$ en tenant compte de critères environnementaux, de sûreté et techniques sur les installations de surface, suivant une logique d'exploitation industrielle. Ils développent une solution «flexible», plus coûteuse, pour élargir les possibilités d'implantation et pouvoir répondre aux demandes d'acteurs politiques locaux : une descenderie (ou tunnel) oblique pour découpler surface et fond, sur un cercle de $5 \mathrm{~km}$ autour du stockage. Le stockage se situe à la frontière des départements de la Meuse et de la HauteMarne, mis en concurrence pour bénéficier des fonds d'accompagnement. Pour que les deux départements reçoivent les fonds, il est ainsi possible de placer les installations de surface et le stockage de part et d'autre de la frontière (contrairement aux puits verticaux initiaux). Les ingénieurs de l'Andra tiennent également compte de contraintes environnementales (zones urbaines, inondables, naturelles protégées, infrastructures de transport).

La troisième étape repose sur une concertation avec des acteurs de la société civile pour définir des « critères d'aménagement du territoire et d'insertion locale ». Afin de préparer en amont cette démarche et établir des conditions de discussion, la directrice générale recrute un responsable des relations publiques et un sociologue, comme responsable des projets de sciences humaines et sociales au sein de l'Andra. Ils définissent un "programme d'information et de consultation», selon lequel l'information et la consultation doivent être intégrées dans la conception du stockage. Selon le responsable des relations publiques, depuis la loi de 2006 , le projet ne peut plus se contenter de répondre à des critères techniques et de sûreté, il doit «être accepté » ${ }^{5}$. Il estime donc nécessaire de construire des relations de « proximité » et de « confiance » ${ }^{6}$ avec les acteurs locaux afin de «faire porter le projet par les élus ». Pour être accompagnée dans cette démarche, la directrice crée en 2007 un comité d'expertise et de suivi de la démarche d'information et de consultation, le Coesdic ${ }^{7}$. Ses membres sont des chercheurs et acteurs opérationnels : Michel Callon (sociologue à l'École des Mines de Paris, membre du conseil scientifique de l'Andra), Anne Bergmans (sociologue à l'université d'Anvers, a participé à la création de partenariats locaux avec

\footnotetext{
5 Entretien avec le chargé des relations publiques, 4 janvier 2013.

6 Entretien avec le chargé des relations publiques, 4 janvier 2013.

7 Première réunion du Coedisc, novembre 2007, site www. andra.fr.
} 
l'Ondraf, équivalent belge de l'Andra), Pierre-Benoît Joly (économiste et sociologue à l'Inra, spécialisé dans les démarches participatives, notamment sur les OGM) et Saida Laârouchi-Engströrm (responsable du Département d'évaluation d'impact environnemental et information publique chez SKB, équivalent suédois de l'Andra).

Ce comité est régulièrement sollicité par la direction pour rendre des avis publics ou des conseils informels sur les sujets politiques et sociaux, et ses avis sont publiés en ligne. Pour le choix d'une zone d'implantation, le Coesdic conseille d'expliciter des choix ouverts et discutables. Il propose que l'Andra prépare une liste « la plus claire et incontestable possible» des options soumises à discussion: elle doit montrer que "les décisions finales ne sont pas encore prises et qu'elles tiendront compte des débats engagés ${ }^{8}$. Début 2009 , l'Andra présente aux acteurs locaux la démarche et les scénarios pour recueillir leurs avis entre mai et octobre 2009. Elle fait ensuite une synthèse de ces échanges: aucun scénario ne fait l'unanimité, mais certains éléments font «consensus». Ainsi, les acteurs locaux considèrent que les critères géologiques et de sûreté doivent être une priorité et ils formulent des demandes d'aménagement du territoire : ils valident le choix de la descenderie, qui permet aux deux départements de bénéficier des fonds. Ils demandent également que les infrastructures favorisent le «développement local» et que les ressources naturelles locales soient préservées.

À partir de ces critères, l'Andra propose une zone de $28 \mathrm{~km}^{2}$; elle montre qu'elle tient compte de demandes sociales et ne provoque pas de mouvements critiques ingérables. En mars 2010, elle est autorisée à mener des investigations géologiques approfondies pour vérifier les qualités du sous-sol et l'absence de failles mineures sur cette zone. La direction de l'Andra parvient donc à concrétiser le projet en associant les acteurs locaux à la réduction des possibles. Cette démarche concertée donne une plus grande légitimité politique au projet, en intégrant certaines critiques de participants, transformés en alliés, sans rediscuter de l'opportunité du projet. Cette démarche est perçue dans le secteur nucléaire comme une réussite, alors qu'aucun opérateur n'a créé de nouveau site nucléaire depuis les années 1980. Elle est ensuite poursuivie en associant les acteurs locaux à la création d'un schéma interdépartemental de développement du territoire en 2013 (eau, électricité, transports, déchets et effluents, emploi et formation) ${ }^{9}$.

Ce dispositif enrôle les acteurs locaux dans l'implantation et la territorialisation du stockage, car cette

\footnotetext{
8 Rapport d'activité du Coesdic, 2008.

9 Schéma interdépartemental de développement du territoire Meuse-Haute-Marne, Projet de Schéma. Document pour le débat public, version 2.6, 21 mars 2013.
}

alliance est essentielle pour lui donner une légitimité politique. Il s'agit de mettre en œuvre un processus maîtrisé de politisation, dans un espace public dépolarisé qui relève davantage d'une controverse en arène (Borraz, 2008). Les acteurs se rassemblent pour échanger et s'accordent sur la nécessité d'élaborer un projet consensuel: les négociations ne portent pas sur l'opportunité du projet, mais sur les modalités de sa réalisation. Le cadrage initial des débats par l'Andra, en définissant ce qui est négociable ou non, permet de réduire les risques de contestation et d'enrôler des acteurs locaux en tenant compte de certaines demandes, au moment particulièrement sensible de la concrétisation du stockage.

\section{Un groupe pluraliste de pilotage pour internaliser les critiques}

Poussant plus loin la logique de participation, la loi de 2006 crée le Plan national de gestion des matières et déchets radioactifs (PNGMDR). La loi donne un cadre formel à un dispositif créé en 2003 par l'Autorité de sûreté nucléaire (ASN) et la Direction générale pour l'énergie et le climat (DGEC, ministère en charge de l'énergie). Il a pour fonction de définir les grandes orientations de cette politique, de fixer un calendrier et de saisir de manière cohérente tous les sujets concernant l'aval de la filière nucléaire. À partir de 2006, ce plan est piloté par un groupe pluraliste : il rassemble opérateurs (EDF, CEA, Areva, Andra), autorités de sûreté, évaluateurs et représentants des ministères. Des associations y participent également (Robin des Bois, Greenpeace, ACRO ${ }^{10}$, FNE $^{11}$, GSIEN $^{12}$, Wise-Paris ${ }^{13}$ ). Dans cette arène pluraliste, les associations peuvent politiser des problèmes et en exiger une prise en charge politique. Elles accèdent aussi à des informations, qu'elles peuvent utiliser dans des espaces publics. Mais ce dispositif permet également de désamorcer certains conflits, à travers un processus de politisation maîtrisé et d'internalisation de critiques.

Cette démarche s'inscrit dans la continuité d'expertises pluralistes des années 1990 (Topçu, 2013), mais elle les dépasse, car il s'agit de fabriquer une politique publique. Malgré les risques de blocage, ce plan gagne une légitimité politique grâce au pilotage pluraliste et, selon le président de l'ASN, il est devenu «quelque chose de tout à fait essentiel » et «d'exemplaire » dans le gouvernement du secteur ${ }^{14}$. Il aurait pu n'être qu'une

\footnotetext{
${ }^{10}$ Association pour le contrôle de la radioactivité de l'Ouest.

11 France Nature Environnement.

12 Groupement de scientifiques pour l'information sur l'énergie nucléaire.

13 World Information Service on Energy.

14 Entretien avec le président de l'ASN, 8 mars 2013.
} 
chambre d'enregistrement ou un lieu d'affrontements polarisés, mais il constitue une arène, les acteurs sont placés dans une situation de négociation et de cogestion de la politique, ce qui atténue leur potentiel critique et dépolarise les controverses. Leurs relations sont différentes de celles mises en scène dans la sphère publique, souvent réduites à un clivage entre pro et anti-nucléaires. Cette arène est le lieu d'un travail d'explicitation des controverses et des positions diverses, au sein du secteur nucléaire et parmi les acteurs critiques. Le groupe est plus ouvert que les dispositifs habituels de pilotage, mais il est non public afin de permettre des échanges libres, car confinés ; il se réunit tous les trois mois. Après des débuts conflictuels, son fonctionnement s'appuie sur des routines mises en place progressivement pour favoriser les discussions entre opérateurs et associations, qui $\mathrm{s}^{\prime}$ accordent pour faire avancer cette politique ${ }^{15}$. Le plan est publié en 2007, actualisé tous les trois ans et évalué par l'Office parlementaire d'évaluation des choix scientifiques et technologiques (OPECST). Initialement, il concernait les déchets, puis la loi de 2006 a intégré l'ensemble des matières radioactives, qui peuvent devenir des déchets si leur valeur d'usage n'est pas démontrée : résidus miniers, combustibles usés, MOX $^{16}$, sources scellées, stockages historiques. Porté par les acteurs réglementaires, les propriétaires de ces matières doivent démontrer qu'il existe des solutions si ces matières sont requalifiées en déchets ${ }^{17}$. Le plan constitue donc un outil essentiel pour gouverner certaines aménités négatives des résidus nucléaires en fonctionnement normal.

À partir d'un inventaire, le groupe de travail identifie les filières nécessaires pour prendre en charge ces objets et il commande des recherches et études aux opérateurs pour définir les solutions qui n'existent pas encore. Selon un directeur de l'Andra, ces commandes sont un «moteur pour faire avancer la politique ${ }^{18}$, pour «poser des jalons » ${ }^{19}$ et s'assurer que les opérateurs «progressent correctement». Les études et recherches constituent donc un outil pour mettre à l'agenda politique certains problèmes, obliger les opérateurs à proposer des solutions et «faire avancer la politique au coup d'après $»^{20}$ : ce plan s'inscrit dans une logique de «science réglementaire» pour orienter la décision

\footnotetext{
${ }^{15}$ Entretien avec le président de l'ASN, 8 mars 2013.

${ }_{17}$ Combustible mélange d'oxydes.

${ }^{17}$ Entretien avec la directrice de la Direction déchets cycle et combustible de l'ASN, 23 avril 2013.

18 Entretien avec le directeur de la Direction maitrise des risques de l'Andra, 6 août 2012.

19 Entretien avec la directrice de la Direction déchets cycle et combustible de l'ASN, 23 avril 2013.

20 Entretien avec le directeur de la Direction maîtrise des risques de l'Andra, 6 août 2012.
}

politique (Jasanoff, 1990). Il est ensuite traduit en décrets pour lui donner une valeur réglementaire et fixer «des programmes de travail cadencés dans le temps $»^{21}$. L'élaboration et l'application de ce plan donnent lieu à de nombreuses négociations. Pour répondre à une injonction d'ouverture du Gouvernement (Topçu, 2013), les acteurs réglementaires ont mis en place un fonctionnement par «cercles $»^{22}$, du plus restreint au plus central, dans lesquels les problèmes et les acteurs circulent. Le plan est d'abord produit dans le cercle le plus restreint, puis, une fois les conflits réglés et le plan stabilisé, il est diffusé dans le cercle plus large et soumis à discussion. Il s'agit donc de suivre le processus définitionnel du plan et les négociations entre sphères confinées et sphères publiques (Gilbert et Henry, 2012).

La rédaction du plan a lieu entre mars et décembre 2012. Les acteurs réglementaires (ASN et DGEC) échangent d'abord de manière unilatérale avec les opérateurs. Puis le contenu est discuté par l'ensemble des opérateurs. Ces derniers font des propositions et, en cas de désaccord, cherchent à convaincre les acteurs réglementaires. Cette phase permet de régler discrètement certains conflits entre les acteurs du secteur et d'assimiler les critiques en amont. La troisième partie du plan est stratégique, car elle présente les " perspectives » et rassemble les demandes d'études et recherches pour définir les objectifs politiques. Après des discussions fin juin, les opérateurs transmettent des propositions rédigées en juillet. Un conflit oppose Andra et producteurs de déchets: il concerne l'attribution des études sur le recyclage des aciers et la valorisation des déchets. Ce marché est en pleine expansion avec le démantèlement des centrales et il s'agit de développer de nouvelles solutions de traitement afin de réduire les déchets stockés en profondeur. L'Andra et les producteurs s'affrontent alors pour obtenir ces études essentielles dans la structuration de futures activités industrielles $^{23}$. Pour atténuer cette concurrence, la DGEC, en tant qu'arbitre, a tenté de les faire coopérer, mais lors du précédent plan les opérateurs ont refusé de travailler ensemble sur ce sujet stratégique économiquement. Néanmoins, après négociations, la DGEC et l'ASN imposent à nouveau cette collaboration dans le futur plan. De la même manière, les études sur l'entreposage en surface des déchets font l'objet de conflits. Dans ce cas, la DGEC et l'ASN optent pour une solution de compromis : les producteurs de déchets conservent les études sur leurs sites, tandis que l'Andra est chargée de l'entreposage en lien avec le stockage géologique.

\footnotetext{
21 Entretien avec le président de l'ASN, 8 mars 2013.

22 Entretien avec le directeur de la Direction maîtrise des risques de l'Andra, 6 août 2012.

23 Entretien avec le directeur de la Direction maîtrise des risques de l'Andra, 6 août 2012.
} 
À l'issue de cette phase, le plan est présenté aux associations en septembre 2012. Lors des phases précédentes, les acteurs réglementaires et les opérateurs ont internalisé les critiques et occulté les enjeux des opérateurs : la concertation porte sur un plan robuste pour limiter les prises aux contestations et cacher les dissensions internes au secteur nucléaire. Lors des séances plénières, les associations s'approprient le registre de l'expertise et mobilisent des arguments scientifiques et techniques pour justifier des demandes politiques. L'ASN et la DGEC retiennent les demandes qu'elles jugent pertinentes et en écartent d'autres. Soutenue par Greenpeace, Robin des Bois, FNE et l'Anccli ${ }^{24}$, l'ACRO tente de faire inscrire dans le plan les rejets d'effluents (dans l'air ou l'eau) ${ }^{25}$ : elle les considère comme des déchets libérés dans l'environnement et pousse plus loin la logique d'élargissement du plan à l'ensemble des matières radioactives. Cependant, les autorités refusent de les inscrire au plan, estimant que les effluents n'en relèvent pas, n'étant pas juridiquement des déchets. Ce désaccord est inscrit dans un encadré afin de le rendre visible, tout en conservant l'adhésion des associations au reste du plan ${ }^{26}$. Un dirigeant de la DGEC explique lors d'une réunion restreinte : «Si on explicite leur position dans le rapport, ils ne pourront pas se désolidariser ${ }^{27}$.» Le plan est ainsi validé dans son intégralité par tous les acteurs, dans une logique de cogestion: il tire sa légitimité de la pluralité des participants à son élaboration.

Le groupe en assure ensuite le pilotage. Les séances plénières sont organisées autour de la présence des associations. Les opérateurs présentent les résultats de leurs études, les associations les discutent, demandent des précisions et soulèvent des problèmes. L'ASN, autorité indépendante, préside les débats et joue le rôle d'arbitre : en cas de désaccord, le président crée un sousgroupe de travail pour réunir les différentes parties et les contraindre à proposer une solution. Ainsi, en juin 2012, FNE et Robin des Bois demandent des données sur les mines d'uranium: elles sollicitent Areva depuis plus d'un an sans succès et demandent à l'ASN d'obliger Areva à leur fournir ces documents ${ }^{28}$. De plus, un désaccord persiste sur la définition du "bruit de fond" radioactif sur les sites miniers. Le président de l'ASN propose alors d'organiser une réunion entre Areva et les

\footnotetext{
$\overline{24}$ Association nationale des comités et des commissions locales d'information.

${ }^{25}$ Observation, réunion $\mathrm{n}^{\mathrm{o}} 37$ du groupe de travail PNGMDR, 14 septembre 2012.

26 Observation, réunions plénières du groupe de travail PNGMDR, 2012.

${ }^{27}$ Observation, réunion restreinte, 12 octobre 2012.

${ }^{28}$ Observation, réunion $\mathrm{n}^{\mathrm{o}} 36$ du groupe de travail PNGMDR, juin 2012.
}

associations pour qu'elles établissent une position consensuelle. Les associations utilisent ainsi cette arène pour faire avancer des sujets bloqués et peuvent ensuite utiliser certaines informations dans des mobilisations publiques.

Cela permet de nuancer les effets de cette « intégration de la critique » (Topçu, 2013), qui viendrait seulement «(re)légitimer les activités nucléaires grâce à la caution associative » et dépolitiser « cette technologie contestée ». Les associations sont loin d'être strictement instrumentalisées par les acteurs nucléaires. Malgré une forte asymétrie, ce dispositif a des effets sur la politisation de certains sujets et la définition de cette politique. Le représentant de Greenpeace explique que les associations ont pu modifier le plan : «La composition pluraliste du groupe de travail représente un atout. En examinant la rédaction des différentes versions de ce plan, nous voyons bien que, dans le premier, les remarques des associations dans l'introduction étaient marginales, séparées du plan. Au cours du temps, nos remarques ont été progressivement intégrées dans le consensus $^{29}$. » Les associations négocient également leur participation et exercent des pressions (notamment lors du débat public de 2013 sur le stockage géologique de déchets). Parallèlement, elles conservent leurs répertoires d'action plus traditionnels (manifestations, actions médiatiques et juridiques).

Néanmoins, ce dispositif a des effets ambigus. Les acteurs nucléaires s'adaptent aux différents cercles pour $\mathrm{y}$ traiter certains sujets, réguler des conflits discrètement et consolider des solutions consensuelles, avant de les publiciser. Ils parviennent à maîtriser ce dispositif de politisation et à limiter les risques de critiques externes, tout en renforçant la légitimité d'un gouvernement « concerté » et «transparent». Ce groupe pluraliste est devenu un lieu de définition et de politisation de problèmes: il n'est pas réductible à une simple assimilation de la critique (Topçu, 2013), sans pour autant correspondre à l'idéal d'une «démocratie technique » (Callon et al., 2001).

\section{Les usages d'un débat raté : utiliser des critiques pour modifier une politique}

Le débat public constitue une troisième modalité d'internalisation des critiques. Le débat de 2013 porte sur les modalités de réalisation du stockage. C'est un moment de politisation et de polarisation de la controverse, parallèlement à l'existence d'arènes pluralistes. Pour les participants, il ne s'agit plus de négocier

\footnotetext{
${ }^{29}$ Observation, audition publique de l'OPECST, 28 février 2013.
} 
des solutions consensuelles, mais de se servir de cette scène pour publiciser leurs positions, convaincre et enrôler (Borraz, 2008). Ce processus est classique et les travaux rendent généralement compte des échanges et de l'évolution des positions des acteurs dans ces espaces publics (Blatrix et al., 2007; Mabi, 2013). Je propose d'adopter un point de vue interne, plus rare, car moins accessible, pour suivre la préparation et la transformation du débat au sein de l'agence, ainsi que son utilisation $a$ posteriori pour modifier le cadre politique du stockage.

Poursuivant sa démarche de concertation, l'Andra organise un débat national en 2013 (obligation de la loi de 2006). Elle rencontre alors des difficultés dans cette démarche, dont la légitimité politique est remise en cause depuis le débat de 2005 sur la gestion des déchets. La Commission particulière du débat avait mis en avant l'entreposage en surface pérennisé comme alternative, les participants au débat ayant privilégié cette option pour des raisons éthiques. Mais il a ensuite été écarté par l'ASN, car il est n'est pas une solution définitive sûre. En 2013, les militants dénoncent cet échec de 2005 et critiquent l'organisation d'un nouveau « faux débat ». La DGEC et l'Andra ont imposé un cadrage préalable au débat: il ne s'agit pas de discuter de l'opportunité du projet (validée par la loi de 2006), mais de négocier les modalités de mise en œuvre. Dans ce contexte tendu, la direction de l'Andra élabore une stratégie de communication intensive pour préparer le débat : les membres de la direction de la communication développent de nombreux supports et sollicitent 2500 acteurs pour qu'ils rédigent des cahiers d'acteurs, ils multiplient les interventions et les réunions publiques pour enrôler des participants.

Initialement, le débat est organisé lors de quatorze réunions publiques hebdomadaires, entre mai et décembre 2013: sur l'inventaire des déchets, le projet et les autres solutions, l'insertion territoriale, la sûreté et la sécurité, la réversibilité, la gouvernance et le coût. La première réunion est organisée le $23 \mathrm{mai}^{30}$ dans la salle des fêtes de Bure. Devant le bâtiment, une manifestation est organisée par une cinquantaine de militants, avec banderoles, chants et tambours. Des gendarmes sont présents à l'extérieur et des agents de sécurité se trouvent à l'intérieur. La salle est principalement occupée par des représentants de l'État, des experts, des opérateurs et des agents de l'Andra occupant les premiers rangs. Des militants sont également présents. Certains, institutionnels, souhaitent participer au débat, pour publiciser leurs positions et recruter des partisans. D'autres ont pour objectif de faire annuler le débat, une « mascarade » dans laquelle ils ont «la parole, mais pas le pouvoir de décision ${ }^{31}$. Le président de la Commission particulière

\footnotetext{
$\overline{30}$ Observation, réunion publique du débat, 23 mai 2013.
}

31 Observation, réunion publique du débat, 23 mai 2013. qui dirige la séance a prévenu qu'au bout d'une demiheure, s'il n'obtenait pas le calme, il suspendrait la séance. Le sachant, les militants empêchent les échanges dès le début de la séance, crient et réclament un référendum départemental. Après quinze minutes, le président suspend la séance et la salle est évacuée. Beaucoup d'acteurs s'attendaient à ce que la première séance soit annulée, pensant que les séances suivantes pourraient avoir lieu. Mais semaine après semaine, le président suspend les séances, alors que la direction de l'Andra estime qu'elles pourraient être maintenues.

Les responsables du débat tentent alors de trouver une solution de remplacement pour que le débat se tienne à l'écart des perturbateurs et pour ne faire participer que les militants institutionnels avec lesquels l'Andra entretient des relations de coopération. La commission du débat public propose de réaliser des « débats contradictoires » filmés et diffusés en direct sur internet et dans des salles. Ces séances sont organisées autour de présentations d'experts pluralistes, puis donnent lieu à des séances de questions-réponses. Un second dispositif est mis en place : une conférence citoyenne. Dix-sept citoyens sont tirés au sort pour être formés pendant deux week-ends par des experts pluralistes ; lors du troisième week-end, ils rédigent un rapport et formulent des recommandations. Ces solutions de remplacement permettent de sortir le débat bloqué d'un espace public trop polarisé pour le replacer dans un espace plus restreint contrôlé : il s'agit d'évacuer et de délégitimer les critiques extrêmes qui remettent en cause l'opportunité du projet et la tenue du débat. Les promoteurs tentent de maitriser davantage le processus de politisation en sélectionnant les critiques compatibles avec l'objectif principal : la construction du stockage géologique.

Au-delà de cet échec relatif, le débat a des effets contradictoires à long terme sur le gouvernement du stockage. Les opérateurs doivent prouver que le débat a permis d'échanger et de modifier certains aspects du projet, pour «consolider la robustesse juridique» du débat $^{32}$. L'Andra accumule les traces de ces échanges (76 000 visites du site internet, 1508 questions, 497 avis, 154 cahiers d'acteurs) ${ }^{33}$ afin de prouver que cette obligation législative est remplie malgré un changement de format. Les dirigeants de l'agence sélectionnent certaines demandes formulées lors du débat pour les assimiler et montrer qu'il a bien eu des effets. Ainsi, certains militants réclament une période d'études plus longue, suivant la position des experts de l'Institut de radiologie et de sûreté nucléaire (IRSN). Ces experts ont participé à la formation du panel de citoyens et ont expliqué que l'Andra devrait mener davantage d'essais

\footnotetext{
Réunion du Coesdic, 13 décembre 2013.

33 Bilan $d u$ débat public, dressé par le président de la Commission nationale du débat public, 12 février 2014.
} 
technologiques, lors d'une période «probatoire»: relâcher le calendrier et créer un « deuxième laboratoire " souterrain avec un "démonstrateur à échelle 1 », pour tester in situ les solutions. Ils ont formulé cette demande à plusieurs reprises sans être entendus par le Gouvernement. Mais cet argument a été repris par certains militants et par la conférence citoyenne qui demandent de desserrer le calendrier et de "réaliser une expérimentation en grandeur réelle $»^{34}$. L'Andra se réapproprie cette stratégie critique dilatoire : de son côté, la création de cette phase «industrielle pilote» lui permettrait de relâcher le calendrier de la loi, jusque-là considéré comme intenable mais obligatoire ${ }^{35}$, et d'euphémiser l'entrée dans la réalisation en multipliant les étapes ${ }^{36}$. La direction choisit donc de mettre en avant cette demande sociale pour montrer que le débat a des effets. Elle soumet ces propositions aux acteurs réglementaires et parlementaires, qui les acceptent et les intègrent dans la loi « réversibilité » du 25 juillet 2016. Les promoteurs du projet ont ainsi sélectionné les critiques à assimiler pour donner une légitimité politique à leur stratégie temporelle.

Néanmoins, cette logique de politisation maîtrisée présente des limites. Malgré ces tentatives de confinement, le débat a favorisé la polarisation de la controverse. Les acteurs critiques les plus radicaux ne reconnaissent pas de légitimité à ce débat euphémisé. Ils adoptent un répertoire d'actions emprunté à des mobilisations sociales contre d'autres projets : au moment du débat, ils créent à Bure une « zone à défendre » sur le modèle de la mobilisation contre l'aéroport de Notre-Dame-desLandes et bénéficient d'une forte médiatisation. À l'été 2016, ils empêchent les travaux de préparation du terrain (eau, électricité, transport). Ces travaux ont lieu avant l'autorisation de construction, ce qui est dénoncé par les militants, qui attaquent juridiquement l'Andra et gagnent temporairement ${ }^{37}$. Depuis, la présence de ces nouveaux militants radicaux a reconfiguré les modes de gouvernement locaux : parallèlement aux relations de coopération qu'entretient l'Andra avec les militants institutionnels, le Gouvernement a recours régulièrement à des interventions de la gendarmerie pour démanteler la zone à défendre et permettre aux travaux d'avancer ${ }^{38}$. Malgré ces tentatives pour maîtriser la politisation, les promoteurs du stockage ne parviennent pas à internaliser ni à

\footnotetext{
$\overline{34}$ Conférence de citoyens, Présentation de l'avis du panel de citoyens, 3 février 2014.

35 Observation, réunion du Coesdic, 7 mars 2012.

36 Observation, réunion du Coesdic, 30 janvier 2014.

37 Tirs croisés d'actions juridiques autour du stockage radioactif de Bure, Le Monde, Pierre Le Hir, 18 août 2016.

38 Évacuation de la ZAD de Bure : mais à qui appartient le Bois-Lejuc?, Sébastien Pommier, 22 février 2018, L'Express.
}

désamorcer toutes les critiques: en distinguant les critiques légitimes de celles qui ne le seraient pas, cette stratégie tend à polariser davantage la controverse autour de la réalisation du stockage.

\section{Conclusion}

Ces trois dispositifs permettent de saisir la stratégie globale d'assimilation des critiques et de transformation $\mathrm{du}$ gouvernement du stockage géologique, et plus largement du secteur nucléaire. L'Andra tente une politisation maîtrisée au moment de la réalisation du stockage: il s'agit d'intégrer certaines critiques afin de réduire les risques de blocage du projet et de le légitimer politiquement. Ce gouvernement pluraliste ne relève pas seulement de l'assimilation de critiques, qui seraient dépolitisées (Topçu, 2013), il se traduit par des changements dans cette politique, la définition de ses objectifs et sa mise en œuvre, à différentes échelles locales et nationales, dans des sphères publiques ou restreintes. Cette politisation contrôlée permet d'enrôler des acteurs critiques et d'adapter le gouvernement d'un projet contesté en fonction de leurs demandes.

Cependant, ces dispositifs participatifs ne permettent pas de rediscuter de l'opportunité de projets considérés comme inéluctables (Blanck, 2016): il s'agit d'en négocier les modalités de mise en œuvre, pas de rouvrir les possibles. Ils ne constituent donc pas de véritables forums hybrides, porteurs de l'idéal d'une démocratie technique (Callon et al., 2001). Ces dispositifs facilitent et encadrent un processus de politisation limité, internalisant certaines critiques et en en évacuant d'autres. Ils donnent une légitimité politique plus forte au projet qui sort renforcé de ces épreuves politiques. Mais ils ont également des effets inattendus, en renforçant la polarisation de cette controverse dans les espaces publics, ce qui se traduit notamment par la création d'une zone à défendre.

Les transformations des modalités de gouvernement de ce projet contesté permettent d'éclairer la politisation d'autres projets d'exploitation du sous-sol : notamment l'implantation de stockages de $\mathrm{CO}_{2}$, le renouveau minier ou encore le stockage géologique d'hydrogène (Arnauld de Sartre, Chailleux, Castro et Garcia, 2019, ce numéro). Face aux contestations, le secteur nucléaire s'est doté dès les années 1970 d'outils de gouvernement de la critique (Topçu, 2013). L'étude de ces dispositifs de gouvernement pluraliste peut éclairer d'autres projets géologiques également confrontés à de fortes contestations et à des processus de politisation plus ou moins maîtrisés. L'articulation entre critiques et politisation est en effet essentielle pour saisir les dynamiques de gouvernement et la territorialisation de ces grands projets techniques à risques. 


\section{Références}

Barthe Y., 2006. Le pouvoir d'indécision. La mise en politique des déchets nucléaires, Paris, Economica, coll. «Études politiques $\gg$.

Blanck J., 2016. Gouverner par le temps : cadrages temporels du problème des déchets radioactifs et construction d'une irréversibilité technique, Gouvernement et action publique, 5, 91-116, https://doi.org/10.3917/gap.161.0091.

Blanck J., 2017. Gouverner par le temps. La gestion des déchets radioactifs en France, entre changements organisationnels et construction de solutions techniques irréversibles (1950-2014). Thèse de Sociologie, Sciences PoInstitut d'études politiques de Paris.

Blatrix C., Blondiaux L., Fourniau J., Lefevre R., Revel M., 2007. Le débat public: une expérience française de démocratie participative, Paris, La Découverte. https:// doi.org/10.3917/dec.blond.2007.01.

Borraz O., 2008. Les politiques du risque, Paris, Presses de Sciences Po.
Callon M., Lascoumes P., Barthe Y., 2001. Agir dans un monde incertain. Essai sur la démocratie technique, Paris, Seuil.

Gilbert Cl., Henry E., 2012. La définition des problèmes publics: entre publicité et discrétion, Revue française de sociologie, 53, 35-59, https://doi.org/10.3917/rfs.531.0035. Hecht G., 2012. Being Nuclear, MIT Press.

Jasanoff Sh., 1990. The Fifth Branch. Science Advisers as Policymakers, Cambridge, Ma, Harvard University Press.

Lagroye J., 2003. La politisation, Paris, Belin.

Mabi C., 2013. Inclusion des publics et matérialité des dispositifs participatifs, Participations, 7, 201-213, https:// doi.org/10.3917/parti.007.0199.

Pierson P., 2000. Increasing returns, path dependence, and the study of politics, The American The American Political Science Review, 94, 2, 251-267. https://doi.org/10.2307/ 2586011.

Topçu S., 2013. La France nucléaire. L'art de gouverner une technologie contestée, Paris, Seuil.

Citation de l'article: Blanck J. Des dispositifs pluralistes sous contrôle : internaliser les critiques pour gouverner le stockage des déchets radioactifs. Nat. Sci. Soc. 29, S33-S42. 